\title{
Efficacy and tolerability of combined medication of two different antimuscarinics for treatment of adults with idiopathic overactive bladder in whom a single agent antimuscarinic therapy failed
}

\author{
Junseok Yi, MD; ${ }^{*}$ Seong Jin Jeong, MD;" Min Soo Chung, MD; Hongzoo Park, MD; ${ }^{+}$Sang Wook Lee, MD; ${ }^{+}$

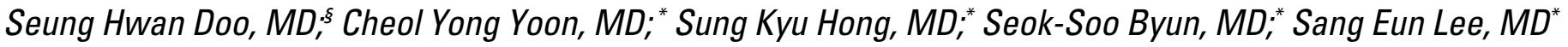

*Department of Urology, Seoul National University Bundang Hospital, Seongnam, Korea; †Department of Urology, Kangwon National University Hospital, Chuncheon, Gangwon-Do, Korea; §Department of Urology, Soonchunhyang University Hospital, Hannam-dong, Yongsan-gu, Seoul, Korea

Cite as: Can Urol Assoc J 2013;7:E88-E92. http://dx.doi.org/10.5489/cuaj.269. Epub 2011 November 2.

\section{Abstract}

Objective: Recent studies have investigated a combination of two antimuscarinics for adult neurogenic bladder managed with clean intermittent catheterization or pediatric refractory overactive bladder $(\mathrm{OAB})$. We assessed the efficacy and tolerability of this strategy in adults with idiopathic OAB.

Methods: We reviewed 49 patients with idiopathic OAB who received combined antimuscarinic medication. Patients had serially received different kinds of antimuscarinics as monotherapy, but wished to take combined medication due to a lack of sufficient subjective improvement in urgency, even with dosage escalation. Efficacy was measured by changes of episodes of urgency, daytime voiding, nocturia and mean voided volume before and after the addition of the second antimuscarinic.

Results: The mean duration of combined medication was 9.3 months. After adding the second antimuscarinic, urgency per day decreased from 3.8 to $1.9(p<0.001)$ and daytime voiding decreased from 10.4 to $7.4(p<0.001)$. The number of nocturia episodes and the mean voided volume also improved, although there was no statistical significance. Efficacy did not differ between the 29 cases, with non-selective and non-selective drugs and 20 cases with non-selective and M3 selective drugs. Thirty-three $(67.3 \%)$ patients reported to have benefited from combined medication. Maximal flow rate and post-void residual volume did not change in either of the sexes. Eleven (22.4\%) patients discontinued the combination due to continued ineffectiveness and dry mouth.

Conclusion: This retrospective study suggests that combined medication can help adults with refractory idiopathic OAB. Combined medication was tolerated in most of our patients.

\section{Introduction}

Antimuscarinics are usually considered first-line therapy for overactive bladder $(\mathrm{OAB})$, along with behavioural modification. ${ }^{1}$ In most cases, antimuscarinics are used as monother- apy and are reported to have higher efficacy with increased dosage. ${ }^{2-4}$ However, for some patients, antimuscarinic medication has a limited effect or falls short due to its associated adverse events. $^{5}$

For refractory OAB symptoms, recent studies have investigated a therapeutic strategy with a combination of two antimuscarinics. Amend and colleagues ${ }^{6}$ and Bolduc and colleagues $^{7}$ reported that the combination of two antimuscarinics could improve episodes of urinary incontinence and urodynamic parameters in adults with neurogenic bladder dysfunction and children with refractory $O A B$.

There have been few reports on the efficacy of combined antimuscarinics for adults with idiopathic OAB other than neurogenic bladder managed with clean intermittent catheterization. In adults with idiopathic $O A B$, the main aim of antimuscarinic therapy is to reduce urgency rather than proven detrusor overactivity (DO), because not all patients have DO or urgency incontinence. In this setting, adverse events on bladder emptying, as well as treatment efficacy, could be a major concern when prescribing a combination of two antimuscarinics.

We performed a retrospective study to evaluate the efficacy and tolerability of a combination of two different antimuscarinics for adults with idiopathic OAB who did not respond to single antimuscarinic and subsequent doubledosage escalation.

\section{Methods}

Between January 2008 and May 2010, we treated 72 adults with a combination of two different antimuscarinics for more than 8 weeks. All of the patients were diagnosed with idiopathic $\mathrm{OAB}$ and demonstrated no prior surgery on lower urinary tract, urinary tract infections and neurological abnormalities based on a detailed history of symptoms, physical examinations and urinalysis. Patients documented the 3-day frequency-volume chart, as well as the Patient's Perception 
of Intensity of Urgency Scale (PPIUS), ${ }^{8}$ at every voiding. The PPIUS is a 5-point scoring system that incorporates the frequency-volume chart and describes the degree of urgency and urgency incontinence felt by the patient. An episode of urgency was defined as PPIUS $\geq 3$ points (severe urgency or urgency incontinence). Patients had serially received different kinds of antimuscarinic agents as monotherapy at the beginning, but wished to take the combined medication due to a lack of sufficient subjective improvement in urgency, even with double-dosage escalation and behavioural modification. Before taking the second drug, all patients underwent cystoscopic examination, urine cytology and urodynamic study (UD-2000, Medical Measures System, The Netherlands) to exclude other bladder diseases, such as interstitial cystitis or carcinoma in situ. Urodynamic study was performed, according to the guidelines of International Continence Society, after the discontinuation of the first drug for 2 weeks. ${ }^{9}$

One physician (SJJ) was in charge of the combination of the two antimuscarinics. The institutional review board of our institution approved this retrospective study protocol. Although selection of the second antimuscarinic drug for combination was at the physician's discretion, a drug that had not been used as monotherapy was preferred as the second antimuscarinic drug. After the addition of the second drug, patients were closely monitored for 2 weeks for possible adverse events with electrocardiogram monitoring, and were then followed-up at 4 weeks and 12 weeks. After that time, patients were followed-up every 3 months with a frequency-volume chart, including PPIUS. The dosage of each combined drug started from a regular recommended dose, and was then increased or decreased based on patient feedback on urgency and adverse events and their frequency-volume chart. To identify any changes in voiding efficiency after combined medication, maximal flow rate (Qmax) and post-void residual (PVR) volume were serially checked. At the last follow-up, we asked our patients: "Have you had any benefit from your treatment?"10

Evaluation of efficacy included changes in urgency, daytime voiding, nocturia and mean voided volume documented on a frequency-volume chart. In addition, we compared the efficacy of combined medication according to the receptor selectivity of administered antimuscarinics. Adverse events were assessed using a questionnaire administered at each visit and graded as none, mild, moderate and severe according to the severity of functional interference.

Collected data were presented as mean \pm standard deviation or percentage. Each parameter was compared before and after the addition of the second drug, using the Wilcoxon signed rank test. Comparisons according to the receptor selectivity of antimuscarinics were performed using the Mann-Whitney $U$ test. Statistical analysis was performed using SPSS version 15.0 (SAS Institute, Cary, NC); $p<0.05$ was considered statistically significant.

\section{Results}

Of the 72 patients, 23 patients took another drug that may have affected voiding simultaneously; they are excluded from the study. In the final analysis, we included 49 adults. We tallied patient demographics and urodynamic findings (Table 1 ). As male patients amounted to only $24.5 \%$ of all patients, we did not differentiate male and female patients when analyzing the treatment efficacy. Mean number of antimuscarinics received as monotherapy was 2.4 and mean monotherapy duration was 7.1 months. Of the patients, 41 had already received oxybutynin as monotherapy. Most of them used propiverine or tolterodine as the last monotherapy drug prior to combination medication.

Of the 49 patients, $95.9 \%$ received both propiverine and other antimuscarinic agent as combination. We recorded the last dosage of each combined drug (Table 2). The mean duration of combined medication was 9.3 months

\section{Table 1. Patient demographics}

\begin{tabular}{|c|c|}
\hline & No. or mean \pm SD \\
\hline No. patients & 49 \\
\hline Age (year) & $61.7 \pm 13.3$ \\
\hline \multicolumn{2}{|l|}{ Sex } \\
\hline Male & 12 \\
\hline Female & 37 \\
\hline \multicolumn{2}{|l|}{ Urodynamic results } \\
\hline $\operatorname{MCC}(\mathrm{ml})$ & $229.1 \pm 109.2$ \\
\hline IDC & 17 \\
\hline IDC volume (ml) & $125.7 \pm 70.9$ \\
\hline IDC pressure $\left(\mathrm{cmH}_{2} \mathrm{O}\right)$ & $38.3 \pm 20.4$ \\
\hline Compliance $\left(\mathrm{ml} / \mathrm{cmH}_{2} \mathrm{O}\right)$ & $34.7 \pm 9.1$ \\
\hline \multicolumn{2}{|l|}{$\mathrm{BOO} *$} \\
\hline Male & 0 \\
\hline Female & 0 \\
\hline \multicolumn{2}{|l|}{ Last antimuscarinic drug as monotherapy } \\
\hline Propiverine $40 \mathrm{mg}, 60 \mathrm{mg}$ & 12,13 \\
\hline Tolterodine $8 \mathrm{mg}$ & 19 \\
\hline Oxybutynin 30 mg, 40mg & 2,3 \\
\hline Monotherapy duration (month) & $7.1 \pm 3.9$ \\
\hline $\begin{array}{l}\text { No. antimuscarinics received as } \\
\text { monotherapy }\end{array}$ & $2.4 \pm 0.8$ \\
\hline \multicolumn{2}{|l|}{$\begin{array}{l}\text { Voiding parameter on the last } \\
\text { monotherapy }\end{array}$} \\
\hline Urgency episodes per $24 \mathrm{hr}$ & $3.8 \pm 2.6$ \\
\hline Daytime voiding episodes per $24 \mathrm{hr}$ & $10.4 \pm 3.6$ \\
\hline Nocturia episodes per $24 \mathrm{hr}$ & $2.4 \pm 1.6$ \\
\hline Voided volume per micturition (ml) & $145.2 \pm 98.5$ \\
\hline $\begin{array}{l}\text { Urgency incontinence episodes per } 24 \mathrm{hr} \\
(\mathrm{N}=15)\end{array}$ & $2.9 \pm 3.2$ \\
\hline \multicolumn{2}{|c|}{$\begin{array}{l}\text { SD: standard deviation; MCC: maximum cystometric capacity; IDC: involuntary detrusor } \\
\text { contraction; BOO: bladder outlet obstruction; *Bladder outlet obstruction was defined } \\
\text { according to the International Continence Society provisional nomogram in males and } \\
\text { Blaivas and Groutz nomogram in females. }\end{array}$} \\
\hline
\end{tabular}


Table 2. The last dosage of combination of two different antimuscarinics according to the patient feedback on urgency and adverse events, and their frequencyvolume chart

\begin{tabular}{|c|c|}
\hline Drug Combinations & $\mathbf{N}$ \\
\hline $\begin{array}{l}\text { Propiverine }\{20 \mathrm{mg}(\mathrm{n}=18), 40 \mathrm{mg}(\mathrm{n}=9)\}+ \\
\text { Tolterodine }\{2 \mathrm{mg}(\mathrm{n}=5), 4 \mathrm{mg}(\mathrm{n}=15) \\
8 \mathrm{mg}(\mathrm{n}=7)\}\end{array}$ & 27 \\
\hline $\begin{array}{l}\text { Propiverine }\{20 \mathrm{mg}(\mathrm{n}=9), 40 \mathrm{mg}(\mathrm{n}=6)\}+ \\
\text { Solifenacin }\{5 \mathrm{mg}(\mathrm{n}=9), 10 \mathrm{mg}(\mathrm{n}=6)\}\end{array}$ & 15 \\
\hline $\begin{array}{l}\text { Propiverine }\{20 \mathrm{mg}(\mathrm{n}=3), 40 \mathrm{mg}(\mathrm{n}=2)\}+ \\
\text { Oxybutynin }\{20 \mathrm{mg}(\mathrm{n}=5)\}\end{array}$ & 5 \\
\hline $\begin{array}{l}\text { Tolterodine }\{8 \mathrm{mg}(\mathrm{n}=2)\}+ \\
\text { Trospium }\{40 \mathrm{mg}(\mathrm{n}=2)\}\end{array}$ & 2 \\
\hline
\end{tabular}

(range: 2-26). After adding the second antimuscarinic drug, episodes of urgency per day decreased significantly from 3.8 to $1.9(p<0.001)$ (Fig. 1$)$ and the mean urge rating per void by the PPIUS showed a significant decrease, from 2.12 to $1.89(p<0.05)$. The number of daytime voiding episodes also decreased by 3.0 per day $(p<0.001$; Fig. 1$)$. However, changes in the number of nocturia episodes and mean voided volume were statistically insignificant $(p>0.05)$ (Fig. 1). Functional bladder capacity, measured by a 3-day frequency-volume chart, improved from $272 \mathrm{~mL}$ to $334 \mathrm{~mL}$ without statistical significance.

Of the 15 patients with urgency incontinence on monotherapy, 11 experienced a reduced degree of incontinence. However, another 4 patients had persistent incontinence after the addition of the second antimuscarinic drug. When comparing the efficacy according to the receptor selectivity of antimuscarinics, no differences were observed between 29 cases with non-selective and non-selective drug and 20 cases with non-selective and M3 selective drug in all parameters (Fig. 1). At the last follow-up, 33 (67.3\%) patients reported to have benefited from the combined medication.

Compared to monotherapy, Qmax and PVR volume did not change significantly in either sex (Table 3). Of the patients, $18(36.7 \%)$ reported no adverse events, $24(49.0 \%)$ had a mild adverse event and 7 (14.3\%) described moderate or severe adverse events. The most commonly reported adverse events were dry mouth, constipation and blurred vision. Notably, none of the patients experienced acute urinary retention or voiding difficulty requiring catheterization or discontinuation of medication. Eleven (22.4\%) patients discontinued the combined medication due to ineffectiveness $(n=7)$ or moderate or severe dry mouth $(n=4)$. Five patients received sacral neuromodulation as further treatment.

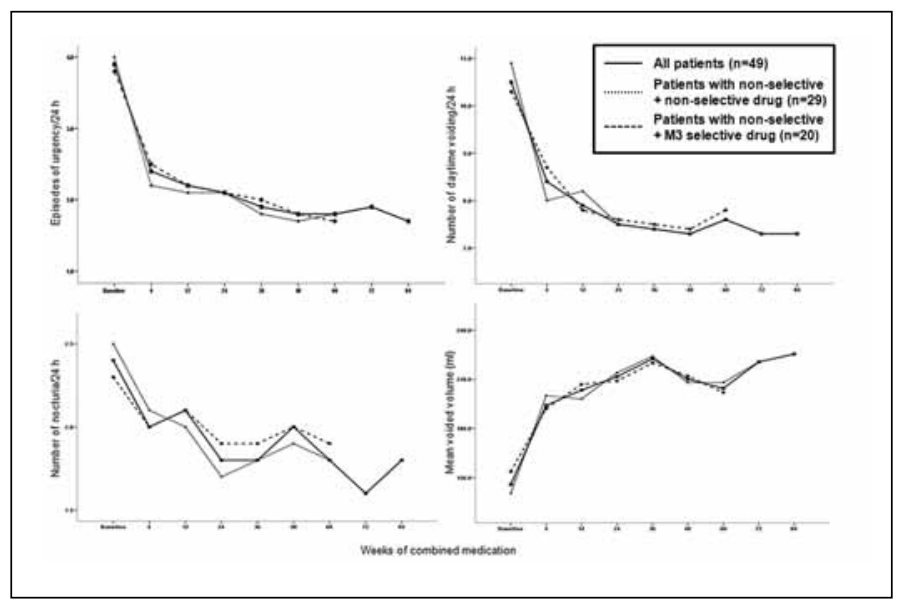

Fig. 1. Changes of episodes of urgency and frequency-volume chart parameters after combined medication. In all of the patients, episodes of urgency and number of daytime voiding episodes per day decreased significantly from baseline (monotherapy) at the last follow-up $(p<0.001$, respectively, Wilcoxon signed rank test). Changes in the number of nocturia episodes and mean voided volume were statistically insignific ant $(p>0.05$, respectively, Wilcoxon signed rank test). When compared according to the receptor selectivity of administered antimuscarinics, no differences were observed between patients with non-selective + non-selective drug and those with non-selective $+\mathrm{M} 3$ selective drug in all parameters (all $p>0.05$, Mann-Whitney $U$ test). Data for analysis were available for 49 patients at baseline, 47 at 12 weeks, 35 at 36 weeks, 19 at 60 weeks, 7 at 84 weeks after combined medication.

\section{Discussion}

A single anticholinergic medication may not be sufficient to induce complete resolution of severe OAB symptoms in up to $30 \%$ of patients. ${ }^{11}$ Therefore, minimally invasive treatments or surgeries have been pursued for patients who do not respond to anticholinergic medication. There have been clinical efforts to maximize the efficacy of antimuscarinics through the combination of two antimuscarinics. ${ }^{6,7}$ Amend and colleagues administered two antimuscarinics to 27 adults with neurogenic bladder dysfunction who had experienced unsatisfactory outcomes with dosage-escalated monotherapy. ${ }^{6}$ Episodes of urgency incontinence, bladder capacity and reflex volume improved after the addition of the second drug. As a result, 22 (85\%) patients were satisfied with the combined antimuscarinic medication. Only 2 patients discontinued combined medication. Bolduc and colleagues administered the combination of two antimuscarinics to 33 children with neurogenic bladder dysfunction or idiopathic OAB. ${ }^{7}$ The mean urodynamic capacity and maximal contraction pressure improved significantly and complete dryness was achieved in 17 (52\%) children. Consequently, a combined medication of two different antimuscarinics may be worth considering for these specific groups before seeking further invasive modalities. 


\begin{tabular}{|c|c|c|c|c|}
\hline & & Baseline (monotherapy) & Combined medication & p-value* \\
\hline \multirow[t]{2}{*}{ Omax (ml/sec) } & Male & $19.2(5.1)$ & $15.5(3.5)$ & 0.060 \\
\hline & Female & $20.7(8.3)$ & $21.5(7.4)$ & 0.901 \\
\hline \multirow[t]{2}{*}{ PVR volume (ml) } & Male & $16.2(10.2)$ & $20.9(10.5)$ & 0.854 \\
\hline & Female & $22.4(25.1)$ & $32.6(29.1)$ & 0.697 \\
\hline
\end{tabular}

*Wilcoxon signed rank test. Data are presented as mean (SD); Omax: maximal flow rate; PVR: post-void residual.

However, the main targeted condition of these studies was refractory neurogenic DO or pediatric idiopathic OAB. Many of these patients were managed with clean intermittent catheterization, where the main aim of combined medication was to improve intravesical pressure to avoid upper urinary tract deterioration and incontinence. Therefore, adverse events on bladder emptying may not be clinically relevant. On the other hand, in adults with idiopathic $O A B$, the main aim of antimuscarinic therapy is to reduce urgency, because not all patients have DO or urgency incontinence. In this setting, adverse events on bladder emptying, as well as treatment efficacy, could be major concerns when prescribing a combination of two antimuscarinics.

We investigated the efficacy and tolerability of combined medication for treatment of adults with idiopathic $O A B$ who had serially received different kinds of antimuscarinic agents as monotherapy at the beginning, but wished to take a combined medication due to a lack of sufficient subjective improvement in urgency, even with double dosage escalation. To the best of our knowledge, there have been few reports on the efficacy and tolerability of combined antimuscarinic medication targeted for adults with idiopathic OAB. In our study, urgency episodes decreased significantly from 3.8 to 1.9 per day and the number of daytime voiding episodes significantly decreased by 3.0 when the second antimuscarinic drug was added. The number of nocturia episodes and the mean voided volume also improved, although there was no statistical significance. The total urine volume per day was about the same (1900-2000 ml) between monotherapy and combined medication. Therefore, patients might have a positive clinical impact on frequency and voided volume per micturition from combined medication. As only $15(30.6 \%)$ patients complained of urgency incontinence on monotherapy, analysis of the changes of urgency incontinence might be underpowered for evaluation of statistical significance; however, $73 \%$ of patients with urgency incontinence experienced a reduced degree of incontinence after the second antimuscarinic drug.

The reason for the enhanced efficacy of combined medication over monotherapy has not been well-understood. The first assumption is the additive effect of regular dosages of two antimuscarinics. ${ }^{11,12}$ However, it seems unreasonable to simply connect our results with a dose-dependent effect because our results demonstrated the enhanced efficacy of combined medication in patients who did not respond to double-dosing monotherapy. Second, different receptor selectivity of two antimuscarinics might be considered. ${ }^{6}$ Expression of the M3 receptor on the bladder is less in a patient with idiopathic DO than in a normal individual, and expression of $\mathrm{M} 2$ or $\mathrm{M} 3$ receptors varies among patients. ${ }^{13}$ If expression of $\mathrm{M} 2$ or $\mathrm{M} 3$ receptors differs among patients, the combination of two antimuscarinics (which have different selectivity toward $\mathrm{M} 2$ or $\mathrm{M} 3$ receptors) will be more effective than a single medication. In this study, however, the efficacy of combined medication did not differ between 29 cases with non-selective and non-selective drug and 20 cases with non-selective and M3 selective drug. Further studies with more patients may determine differences in the efficacy of various combinations according to M3 receptor selectivity. Third, a synergistic effect of two different antimuscarinics may be considered. From the physiologic aspect, each receptor in the human body is known to trigger chain reactions or amplify the response through mutual feedback, rather than perform independent functions. ${ }^{14}$ Therefore, a synergistic effect of each drug on different muscarinic receptors might be responsible for the enhanced efficacy of our results. Finally, the different rate of metabolism of antimuscarinics in each patient might be one explanation. ${ }^{6}$

The greatest concern may involve adverse events and tolerability in the clinical use of this two-antimuscarinic strategy for patients with idiopathic OAB. In our study, however, Qmax and PVR volume were not different between monotherapy and combined medication in both sexes. Although $31(63.3 \%)$ patients reported adverse events, most adverse events were mild and discontinuation of combined medication due to these adverse events occurred in only 4 (8.2\%) patients. Bolduc and colleagues attributed the low number of adverse events with combined medication to differences in antimuscarinic metabolites and their target receptors among drugs. ${ }^{7}$ However, because studies of combined medication of two anticholinergics, ${ }^{6,7}$ including our study, have been conducted on patients who had not experienced severe adverse events in prior monotherapy, clinical use of combined medication should be implemented with caution in general $O A B$ populations. On the other hand, patients might experience fewer adverse events and enhanced tolerability in combination medication than expected, because 96\% (47/49) of patients received propiverine as one of the combination drugs, and only $10 \%(5 / 49)$ received oxybutynin as one of the combination drugs. Previous studies have demonstrated 
that propiverine is as effective as oxybutynin and is better tolerated than oxybutynin in adults ${ }^{15}$ and in children ${ }^{16}$ with OAB symptoms. Therefore, we might obtain different results with regard to side effects and tolerability, if oxybutynin was used more frequently in combination medication.

The present study had several limitations. First, the decision to switch from monotherapy to combined medication was at the patient's discretion rather than objective criteria. Second, although most patients (95.9\%) received both propiverine and other antimuscarinic agent as combination, antimuscarinics used in combination were heterogeneous among patients. These limitations are attributed to the retrospective design of the study and should be dealt with in a prospective study. In combination medication, propiverine was preferred over oxybutynin due to the concern that adverse events might increase and drug tolerability might be decreased following combination medication. However, along with the previous studies, ${ }^{6,7}$ our results might lay the groundwork for further studies on dual antimuscarinic medication in adults with idiopathic OAB. Third, clinical effects of combined medication might not be derived from the combination, but from the switching in some patients because there was no placebo arm and not all patients had received each drug used in combination as monotherapy. In clinical practice, most urologists would try another anticholinergic for patients who fail one anticholinergic before considering a combination of two antimuscarinics. ${ }^{17-19}$ These studies also did not include a placebo arm or direct comparator, and, therefore, might be open to physician and patient bias. Further research should encompass this issue to ensure the efficacy of simply changing anticholinergics or combined medication in real practice. Last, we did not perform urodynamic studies in patients after the addition of the second antimuscarinic agent. We think that follow-up urodynamic studies should be conducted to determine actual changes of bladder storage and voiding function.

\section{Conclusion}

This retrospective study suggests that the combination of two antimuscarinics can improve symptoms of urgency and frequency in adults with idiopathic OAB who did not respond to a previous single medication with no significant increase of adverse events. When antimuscarinic monotherapy is not effective, the combined medication of two antimuscarinics may be another option before considering further treatments, such as minimally or classic invasive treatments.

Acknowledgments: The authors would like to acknowledge Dr. Daesung Kim and Dr. Wonki Lee for their contributions to this study as advisor for medical writing.
Competing interests: None declared.

This paper has been peer-reviewed.

\section{References}

1. Andersson KE. Antimuscarinics for the treatment of overactive bladder. Lancet Neurol 2004;3:46-53.

2. Van Kerrebroeck PE, Amarenco G, Thüroff JW, et al. Dose ranging study of tolterodine in patients with detrusor hyperreflexia. Neurourol Urodyn 1998;17:499-512.

3. Van Kerrebroeck $\mathrm{P}$, Kreder $\mathrm{K}$, Jonas $\mathrm{U}$, et al. Tolterodine once-daily: superior efficacy and tolerability in the treatment of the overactive bladder. Urology 2001;57:414-21.

4. Appell RA. The newer antimuscarinic drugs: bladder control with less dry mouth. Cleve Clin J Med 2002;69:761-9.

5. Madersbacher H. Oral anticholinergics in overactive bladder. Urologe A 2006;45:830-4.

6. Amend B, Hennenlotter J, Schäfer T, et al. Effective treatment of neurogenic detrusor dysfunction by combined high-dosed antimuscarinics without increased side-effects. Eur Urol 2008;53:1021-8.

7. Bolduc $S$, Moore K, Lebel $S$, et al. Double anticholinergic therapy for refractory overactive bladder. J Urol 2009;182:2033-8

8. Cardozo L, Hessdörfer E, Milani R, et al. Solifenacin in the treatment of urgency and other symptoms of overactive bladder: results from a randomized, double-blind, placebo-controlled, rising-dose trial. BJU Int 2008:102:1120-7.

9. Schäfer W, Abrams P, Liao L, et al. International Continence Society: Good urodynamic practices: uroflowmetry, filling cystometry, and pressure-flow studies. Neurourol Urodyn 2002;21:261-74.

10. Pleil AM, Coyne KS, Reese PR, et al. The validation of patientrated global assessments of treatment benefit, satisfaction, and willingness to continue-the BSW. Value Health 2005;8:S25-S34.

11. MacDiarmid SA. Overactive bladder: improving the efficacy of anticholinergics by dose escalation. Curr Urol Rep 2003;4:446-51.

12. Horstmann M, Schaefer T, Aguilar Y, et al. Neurogenic bladder treatment by doubling the recommended antimuscarinic dosage. Neurourol Urodyn 2006;25:441-5.

13. Mansfield KJ, Liu L, Moore KH, et al. Molecular characterization of M2 and M3 muscarinic receptor expression in bladder from women with refractory idiopathic detrusor overactivity. BJU Int 2007;99:1433-8.

14. Andersen ME, Barton HA. Biological regulation of receptor-hormone complex concentrations in relation to dose-response assessments for endocrine-active compounds. Toxicol Sci 1999; 48:38-50.

15. Madersbacher $H$, Halaska $M$, Voigt $R$, et al. A placebo-controlled, multicentre study comparing the tolerability and efficacy of propiverine and oxybutynin in patients with urgency and urge incontinence. BJU Int 1999;84:646-51.

16. Alloussi S, Mürtz G, Braun R, et al. Efficacy, tolerability and safety of propiverine hydrochloride in comparison to oxybutynin in children with urge incontinence due to overactive bladder: Results of a multicentre observational cohort study. BJU Int 2010;106:550-6.

17. Swift SE, Siami P, Forero-Schwanhaeuser $S$. Diary and patient-reported outcomes in patients with severe overactive bladder switching from tolterodine extended release $4 \mathrm{mg} /$ day to solifenacin treatment: An open-label, flexible-dosing, multicentre study. Clin Drug Investig 2009;29:305-16.

18. Chancellor MB, Zinner N, Whitmore $\mathrm{K}$, et al. Efficacy of solifenacin in patients previously treated with tolterodine extended release $4 \mathrm{mg}$ : results of a 12-week, multicenter, open-label, flexible-dose study. Clin Ther 2008;30:1766-81.

19. Zinner N, Noe L, Rasouliyan L, et al. Impact of solifenacin on resource utilization, work productivity and health utility in overactive bladder patients switching from tolterodine ER. Curr Med Res Opin 2008;24:1583-91.

Correspondence: Dr. Seong Jin Jeong, Department of Urology, Seoul National University Bundang Hospital, 300 Gumi-dong, Bundang-gu, Seongnam, Korea 463-707; fax: +82-31-787-4057; uroisj@empal.com 